\title{
High flow nasal cannula versus non-invasive ventilation in severe asthma: two years prospective observational study.
}

\author{
Carolina Solé-Delgado ${ }^{1}$, Alberto García-Salido ${ }^{1}$, Ainhoa Gochi-Valdovinos ${ }^{2}$, Anthony \\ González-Brabin², Maria García ${ }^{2}$, Amelia Martínez de Azagra-Garde ${ }^{3}$, Inés Leoz-Gordillo², \\ Mabel Iglesias-Bouzas ${ }^{4}$, Marta Cabrero-Hernández² ${ }^{2}$ José Luis Unzueta-Roch ${ }^{2}$, Gema De \\ Lama Caro-Patón ${ }^{2}$, and Montserrat Nieto ${ }^{2}$ \\ ${ }^{1}$ Hospital Infantil Universitario Niño Jesús Servicio de Cuidados Intensivos Pediátricos \\ ${ }^{2}$ Hospital Infantil Universitario Nino Jesus \\ ${ }^{3}$ Hospital Infantil Universitario Niño Jesús \\ ${ }^{4}$ Hospital Infantil Universitario Niño Jesús. Madrid, Spain.
}

March 19, 2021

\begin{abstract}
Background: in recent years, High Flow Nasal Cannula (HFNC) has been considered an alternative to non-invasive mechanical ventilation (NIMV) in severe asthma respiratory management in children. Objective: to describe the use of HFNC in children with severe asthma admitted to pediatric critical care unit (PICU). To compare its clinical characteristic and evolution with those receiving NIMV or other respiratory support. Methods: prospective observational study done in children admitted to PICU with severe asthma (October 2017 to October 2019). Data collected: epidemiological, clinical, respiratory support, thorax x-ray, pharmacological treatments and days of admission. Patients were divided into groups: 1) Only HFNC 2) HFNC and NIMV, and 3) Only NIMV. Results: Seventy-six patients included, 39 girls. The median age was two years and one month (range 160). The median pulmonary score was 5 (range 7). PICU admission lengths a median of 3 days (range 9), hospital 6 days (range 23). There were no epidemiological or clinical differences between groups. Children with only HNFC showed a shorter time of PICU days (p 0,025) and none of them required NIMV. In the group receiving both modalities, NIMV was used first and then HFNC in all cases. Children with HFNC showed higher SaO2/FiO2 ratio $(\mathrm{p}=0,025)$ and lower PCO2 level $(\mathrm{p}=0,032)$. There were no deaths. Conclusions: in our study the HFNC did not require escalation to NIMV and did not increase the length of PICU or hospital days. Normal initial blood gases and absence of high oxygen requirements were useful to select responders to HNFC.
\end{abstract}

High flow nasal cannula versus non-invasive ventilation in severe asthma: two years prospective observational study.

Carolina Solé Delgado ${ }^{1}$, Alberto García-Salido MD, $\mathrm{PhD}^{2}$, Ainhoa Gochi Valdovinos ${ }^{1}$, Anthony González Brabin $^{1}$, María Ángeles García-Teresa $\mathrm{MD}^{1}$, Amelia Martínez de Azagra-Garde MD ${ }^{1}$, Inés Leoz-Gordillo $\mathrm{MD}^{1}$, María Isabel Iglesias-Bouzas $\mathrm{MD}^{1}$, Marta Cabrero-Hernández $\mathrm{MD}^{1}$, José Luis Unzueta-Roch $\mathrm{MD}^{1}$, Gema De Lama Caro-Patón MD ${ }^{1}$, Montserrat Nieto-Moro MD ${ }^{1}$

${ }^{1}$ Pediatric Critical Care Unit, Hospital Infantil Universitario Niño Jesús, Madrid, Spain.

${ }^{2}$ Corresponding author : Alberto García-Salido, MD, PhD, Pediatric Intensive Care Unit. Hospital Infantil Universitario Niño Jesús. Avenida Menéndez Pelayo 65, Madrid, Spain. +34 915035900. https://orcid.org/0000-0002-8038-7430 Email;citopensis@yahoo.es.

No funding. 
The authors have disclosed that they do not have any potential conflicts of interest.

Keywords : asthma, pediatric critical care, children, high flow nasal cannula, non-invasive ventilation

Background : in recent years, High Flow Nasal Cannula (HFNC) has been considered an alternative to non-invasive mechanical ventilation (NIMV) in severe asthma respiratory management in children.

Objective : to describe the use of HFNC in children with severe asthma admitted to pediatric critical care unit (PICU). To compare its clinical characteristic and evolution with those receiving NIMV or other respiratory support.

Methods : prospective observational study done in children admitted to PICU with severe asthma (October 2017 to October 2019). Data collected: epidemiological, clinical, respiratory support, thorax x-ray, pharmacological treatments and days of admission. Patients were divided into groups: 1) Only HFNC 2) HFNC and NIMV, and 3) Only NIMV.

Results : Seventy-six patients included, 39 girls. The median age was two years and one month (range 160). The median pulmonary score was 5 (range 7). PICU admission lengths a median of 3 days (range 9), hospital 6 days (range 23). There were no epidemiological or clinical differences between groups. Children with only HNFC showed a shorter time of PICU days (p 0,025) and none of them required NIMV. In the group receiving both modalities, NIMV was used first and then HFNC in all cases. Children with HFNC showed higher $\mathrm{SaO}_{2} / \mathrm{FiO}_{2}$ ratio $(\mathrm{p}=0,025)$ and lower $\mathrm{PCO}_{2}$ level $(\mathrm{p}=0,032)$. There were no deaths.

Conclusions: in our study the HFNC did not require escalation to NIMV and did not increase the length of PICU or hospital days. Normal initial blood gases and absence of high oxygen requirements were useful to select responders to HNFC.

\section{INTRODUCTION}

Asthma exacerbation is a frequent cause of pediatric intensive care unit (PICU) admission ${ }^{1-3}$. Its management includes pharmacology treatment and respiratory support with invasive mechanical ventilation as the last step. This modality, even though useful, may bring complications ${ }^{4}$ so other forms of respiratory support are discussed and debated as first-line approaches ${ }^{5-7}$. Nowadays, one of these options is high flow nasal cannula (HFNC).

The HFNC application has quickly spread in pediatrics due to its simplicity and comfort ${ }^{8-10}$. Its use of has been describe in neonatal wards ${ }^{11,12}$, emergency department ${ }^{13-15}$, hospital units ${ }^{11-17}$, transport $^{18}$ and intensive care units ${ }^{19-21}$. In PICU the use of HNFC has been described with limited data about its usefulness or security in bronchiolitis ${ }^{22-24}$ or asthma ${ }^{20,21,25}$. It is a controversial approach because there is no strong evidence to recommend it. It's a concern that HFNC could delay the start of other ventilatory strategies with proven efficacy like non-invasive mechanical ventilation (NIMV).

Consider this, in this prospective observational monocentric study, we describe and analyse the respiratory therapies applied to children with PICU admission because of asthma exacerbation. The primary objective was to analyse and describe the clinical and epidemiological characteristics of these cases. As a secondary objective, we evaluated the use of HFNC in these children and compared to other ways of respiratory support.

\section{METHODS}

\section{Design}

Observational, prospective, longitudinal study on a tertiary PICU (14 bed with and a mean of 800 admissions per year). It was done in children with asthma exacerbation and PICU admission (from October 2017 to October 2019). The ethics committee from Hospital Infantil Universitario Niño Jesús approved it. The data were obtained from clinical registers and was performed following the principles of the Declaration of Helsinki. The authors assume responsibility for the accuracy and completeness of the data and analyses and for the fidelity of the report. The attending in charge decided the clinical management based on the clinical protocols applied in our unit. Children with severe comorbidities or previous disease were excluded. 


\section{Inclusion criteria}

Patients were enrolled if they fulfilled the following conditions:

- Less than 18 years old.

- Acute exacerbation of asthma was considered as an acute episode of increased work of breathing with wheeze and prolonged expiratory phase in a child with similar previous episodes. There should not be improved despite optimal measures in the emergency department or ward. These measures must include: optimised salbutamol nebulisation, $2 \mathrm{mg} / \mathrm{kg}$ intravenous glucocorticoids (GC, methylprednisolone) and rescue therapy with magnesium sulphate. Severe asthma was considered if $\mathrm{SatO}_{2}<90 \%$ with gasping, bradypnea, apnea, cyanosis or altered mental status

- Non-severe comorbidities or previous diseases except asthma.

- No acute bronchiolitis criteria defined as the onset of wheezing before 24 months of age in patients presenting viral lower respiratory infection on physical examination with no other explanation for the wheezing.

\section{Respiratory support applied}

No invasive mechanical ventilation (NIV): BIPAP Vision V60@ (Respironics Philips) with full face or oronasal mask. Modalities: continuous positive pressure (CPAP) and bi-level positive pressure (BiPAP). Initially, the CPAP was started at 5-6 $\mathrm{cmH}_{2} \mathrm{O}$. In case of BiPAP, the Inspiratory Positive Airway Pressure was set at beginning at 8-10 $\mathrm{cmH}_{2} \mathrm{O}$ and the End-positive Airway Pressure at 5-6 $\mathrm{cmH}_{2} \mathrm{O}$. Inspiratory and expiratory pressure was titrated in increments of $2 \mathrm{cmH}_{2} \mathrm{O}$ based on tidal volume, continuous pulse oximetry, work of breathing, respiratory rate and subject-ventilator synchrony. The fraction of inspired oxygen $\left(\mathrm{FIO}_{2}\right)$ was titrated to maintain a $\mathrm{SpO}_{2}>92 \%$.

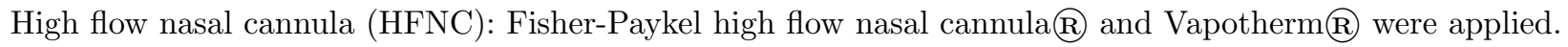
A cannula of suitable size, an appropriate circuit, humidifier and air/oxygen. Cannula size was selected based on subject weight Flow rates were initiated at $0,5-1 \mathrm{~L} / \mathrm{kg} / \mathrm{min}$. The fraction of inspired oxygen (FIO2) was titrated to maintain a $\mathrm{SpO}_{2}>92 \%$.

\section{Collected data}

Patient data were collected anonymously, once hospital admission is over and without intervening in everyday clinical decisions.

Demographic characteristics (age in months and gender).

Clinical data: respiratory rate on admission $(\mathrm{RR})$, presence and characteristics of wheezing, pulmonary score (PS), $\mathrm{SatO}_{2} / \mathrm{FiO}_{2}$ ratio, venous blood gas values at admission $\left(\mathrm{pH}, \mathrm{PCO}_{2}, \mathrm{HCO}_{3}\right.$ ), pharmacological treatment received (bronchodilators, corticosteroids, antibiotic therapy, magnesium sulfate), type and days of ventilatory support, length of stay in the PICU, and the total length of hospital stay.

After data collection, three study groups were created: 1) "Only HFNC", 2) "NIMV and HFNC" and 3) "Only NIV".

\section{Statistical analysis}

The data analysis was performed using the SPSS(r) statistical package (version 21.0; IBM Company(r); New York, United States). The homogeneity of the demographic variables and other clinical parameters were analysed at the beginning of the study (having a non-normal distribution) and compared among groups. The description will be made using the quantitative median and range, as well as the absolute frequency and relative frequency of the qualitative variables. For the analysis of the characteristics of the three treatment groups, a Kruskal Wallis test was performed in the case of quantitative variables and Fisher's exact test in the case of dichotomous variables.

\section{Results}


During the study, 86 children were recruited. Four were excluded due to significant comorbidities (cerebral palsy, leukaemia, mucolipidosis and bronchopulmonary dysplasia with home oxygen) and 6 due to iatrogenic bronchospasm (after intubation, after bronchoscopy or sedation, Figure 1).

Epidemiological data, severity on admission, analytical data and pharmacological therapies

There were no differences between groups in sex, age, $\mathrm{PS}, \mathrm{pH}$ at admission, $\mathrm{HCO}_{3}$ value at admission, $\mathrm{RR}$, or administration of GC. Fractionally nebulised bronchodilators were used in all patients during admission. Data are summarised in Table 1. Differences were observed in the frequency of performing chest radiography, empirical antibiotic therapy, continuous salbutamol, magnesium sulfate. All of these being less frequent in the case of receiving only HFNC (Table 2).

Evolution and respiratory assistance

The days of PICU admission were lower in the group with only-HFNC (p 0.025). The $\mathrm{SatO}_{2} / \mathrm{FiO}_{2}$ was lower in the only-NIMV group and higher in the only-HFNC group (p 0.026). The $\mathrm{PCO}_{2}$ was more elevated in the NIMV-only group (p 0.032). Also, the median number of days with HFNC is lower in the only- HFNC group than in the one in which HFNC is combined with NIMV (2 days versus 3; Table 3 ). None of the patients who received HFNC initially required NIMV later. There were no intubations or deaths.

\section{DISCUSSION}

In our study, HFNC was a proper respiratory management for patients admitted to PICU because of asthma. We observed that there was no difference in PS at admission between children which only received HNFC or those with NIMV support. Children with only HNFC received less pharmacological therapies and have fewer days of hospitalization. Finally, they did not require escalating therapeutic measures the days of PICU admission were lower. We observed that lower $\mathrm{SatO}_{2} / \mathrm{FiO}_{2}$ and higher $\mathrm{PCO}_{2}$ at admission were related to the use of NIMV.

The HFNC delivers a flow of warm and humidified air, with a variable oxygen fraction (between 0,21-1) and a flow between 2 and 60 litres. From a theoretical point of view, the HFNC reduces the oropharyngeal dead space, reduces $\mathrm{CO}_{2}$ rebreathing ${ }^{26}$, improves mucociliary clearance ${ }^{27}$ and generate an end positive pressure in the airway up to $6 \mathrm{cmH}_{2} \mathrm{O}$ during expiration ${ }^{17,28,29}$. The growing interest in the use of HFNC as respiratory support derives from these properties added to a higher comfort for the patient ${ }^{24,29}$. It also does not need synchronisation with patient breathing and requires less nursing care (versus a NIMV device). The HFNC would help the respiratory muscles to overcome the auto-peep characteristic of obstructive pathologies. Also, this constant flow may facility the renewal of nasopharyngeal air that would contribute to improving $\mathrm{CO}_{2}$ washing and oxygenation ${ }^{17}$.

As previously said, asthma is one of the leading causes of PICU admission. The evidence about the utility of HFNC as optimal respiratory support is scarce. Recently, Ramnarayan et al. carried out a pilot study to assess it through a multicenter and randomised clinical trial. They found that it was common to switch treatment from HFNC to CPAP. Also, HFNC patients experienced fewer ventilator-free days at day $28^{21}$. Added to this, an observational study in 42 asthmatic children by Pilar et al.concluded that initial support with HFNC was no optimal and delay support with $\mathrm{NIMV}^{20}$. In our prospective observational study, the HFNC was the most frequent respiratory assistance applied. In $74 \%$ of patients, the HFNC was their first and only support. None of these children needs to escalate to NIMV. In the rest cases, the HFNC was always used as de-escalation of NIV.

About this absence of necessity to switch from HFNC to NIMV, we must do some commentaries. As can be seen in our series children with higher $\mathrm{PCO}_{2}$ and lower $\mathrm{SatO}_{2} / \mathrm{FiO}_{2}$ received NIMV as first support. Furthermore, there is a tendency to use HFNC in older patients (median age 25 versus 14 months in the case of NIV, $\mathrm{p}>0.05$ ). There were also differences among groups regarding chest radiography, initiation of empirical antibiotic therapy, administration of magnesium sulfate, and continuous nebulisation of salbutamol. Although we did not find differences in PS we thing that children with NIMV support were more severe at 
PICU admission. As explained previously the management was not protocolised. The attending experience influenced the respiratory assistance chosen and act as a selection bias that for sure influences our results.

About the days of PICU or hospital admission, they were no longer in HNFC group that in others (Table 3 ). These findings are contrary to what has been described in other studies ${ }^{21}$. This observation may be related to this selection bias in which HNFC will be effective.

This study has several limitations. It is a single-centre study. Patients under the age of two are included. Although those with acute bronchiolitis diagnosis were excluded, this fact should be taken into account. It is also observed that this was the age group in which the need for non-invasive was higher. The choice between different therapeutic options was influenced by the know-how of the physician in charge of each patient. This non-protocolised approach influenced the results through a selection bias about the utility of HFNC. It also informs about the importance of clinical experience to ensure the success of this respiratory approach.

In summary, in our study, the HFNC was a safe approach that did not require escalation to NIV. It did not increase the length of hospital admission. Initial blood gases and the absence of high oxygen requirements on admission could be helpful to select children that will be good responders to HNFC. The external validation of our results is complex. Randomised and multicenter clinical trials are needed to verify this data.

\section{CONFLICT OF INTEREST:}

This study has not been funded. The authors declare that they have no conflicts of interest.

\section{Figure legends.}

Figure 1. Flowchart of patient inclusion and exclusion.

\section{Bibliography}

1. Asher I, Pearce N. Global burden of asthma among children. The international journal of tuberculosis and lung disease: the official journal of the International Union against Tuberculosis and Lung Disease. 2014;18(11):1269-1278.

2. Gupta D, Nath A, Agarwal R, Behera D. A prospective randomized controlled trial on the efficacy of noninvasive ventilation in severe acute asthma. Respiratory care.2010;55(5):536-543.

3. Papi A, Brightling C, Pedersen SE, Reddel HK. Asthma. Lancet (London, England).2018;391(10122):783800.

4. Maggiore SM, Idone FA, Vaschetto R, et al. Nasal high-flow versus Venturi mask oxygen therapy after extubation. Effects on oxygenation, comfort, and clinical outcome.American journal of respiratory and critical care medicine.2014;190(3):282-288.

5. Bratton SL, Newth CJ, Zuppa AF, et al. Critical care for pediatric asthma: wide care variability and challenges for study. Pediatric critical care medicine : a journal of the Society of Critical Care Medicine and the World Federation of Pediatric Intensive and Critical Care Societies. 2012;13(4):407-414.

6. Kao CC, Jain S, Guntupalli KK, Bandi V. Mechanical ventilation for asthma: a 10-year experience.The Journal of asthma : official journal of the Association for the Care of Asthma. 2008;45(7):552-556.

7. Gehlbach B, Kress JP, Kahn J, DeRuiter C, Pohlman A, Hall J. Correlates of prolonged hospitalization in inner-city ICU patients receiving noninvasive and invasive positive pressure ventilation for status asthmaticus. Chest.2002;122(5):1709-1714.

8. Baudin F, Gagnon S, Crulli B, Proulx F, Jouvet P, Emeriaud G. Modalities and Complications Associated With the Use of High-Flow Nasal Cannula: Experience in a Pediatric ICU.Respiratory care. 2016;61(10):1305-1310.

9. Spentzas T, Minarik M, Patters AB, Vinson B, Stidham G. Children with respiratory distress treated with high-flow nasal cannula. Journal of intensive care medicine.2009;24(5):323-328. 
10. Testa G, Iodice F, Ricci Z, et al. Comparative evaluation of high-flow nasal cannula and conventional oxygen therapy in paediatric cardiac surgical patients: a randomized controlled trial. Interactive cardiovascular and thoracic surgery. 2014;19(3):456-461.

11. Ojha S, Gridley E, Dorling J. Use of heated humidified high-flow nasal cannula oxygen in neonates: a UK wide survey. Acta paediatrica (Oslo, Norway : 1992).2013;102(3):249-253.

12. Wilkinson D, Andersen C, O'Donnell CP, De Paoli AG, Manley BJ. High flow nasal cannula for respiratory support in preterm infants. The Cochrane database of systematic reviews. 2016;2:Cd006405.

13. Long E, Babl FE, Duke T. Is there a role for humidified heated high-flow nasal cannula therapy in paediatric emergency departments? Emergency medicine journal : EMJ. 2016;33(6):386-389.

14. Slain KN, Shein SL, Rotta AT. The use of high-flow nasal cannula in the pediatric emergency department. Jornal de pediatria. 2017;93 Suppl 1:36-45.

15. Wang J, Lee KP, Chong SL, Loi M, Lee JH. High flow nasal cannula in the emergency department: indications, safety and effectiveness. Expert review of medical devices. 2018;15(12):929-935.

16. Gonzalez Martinez F, Gonzalez Sanchez MI, Toledo Del Castillo B, et al. Treatment with high-flow oxygen therapy in asthma exacerbations in a paediatric hospital ward: Experience from 2012 to 2016. Anales de pediatria (Barcelona, Spain : 2003). 2019;90(2):72-78.

17. Milesi C, Boubal M, Jacquot A, et al. High-flow nasal cannula: recommendations for daily practice in pediatrics. Annals of intensive care. 2014;4:29.

18. Schlapbach LJ, Schaefer J, Brady AM, Mayfield S, Schibler A. High-flow nasal cannula (HFNC) support in interhospital transport of critically ill children. Intensive care medicine. 2014;40(4):592-599.

19. Coletti KD, Bagdure DN, Walker LK, Remy KE, Custer JW. High-Flow Nasal Cannula Utilization in Pediatric Critical Care. Respiratory care. 2017;62(8):1023-1029.

20. Pilar J, Modesto IAV, Lopez-Fernandez YM, Lopez-Macias O, Garcia-Urabayen D, Amores-Hernandez I. High-flow nasal cannula therapy versus non-invasive ventilation in children with severe acute asthma exacerbation: An observational cohort study. Medicina intensiva. 2017;41(7):418-424.

21. Ramnarayan P, Lister P, Dominguez T, et al. FIRST-line support for Assistance in Breathing in Children (FIRST-ABC): a multicentre pilot randomised controlled trial of high-flow nasal cannula therapy versus continuous positive airway pressure in paediatric critical care. Critical care (London, England). 2018;22(1):144.

22. Bermudez Barrezueta L, Garcia Carbonell N, Lopez Montes J, et al. High flow nasal cannula oxygen therapy in the treatment of acute bronchiolitis in neonates.Anales de pediatria (Barcelona, Spain : 2003). 2017;86(1):37-44.

23. Hilliard TN, Archer N, Laura H, et al. Pilot study of vapotherm oxygen delivery in moderately severe bronchiolitis. Archives of disease in childhood.2012;97(2):182-183.

24. Mayfield S, Bogossian F, O'Malley L, Schibler A. High-flow nasal cannula oxygen therapy for infants with bronchiolitis: pilot study. Journal of paediatrics and child health. 2014;50(5):373-378.

25. Mayfield S, Jauncey-Cooke J, Bogossian F. A case series of paediatric high flow nasal cannula therapy. Australian critical care : official journal of the Confederation of Australian Critical Care Nurses. 2013;26(4):189-192.

26. Roca O, Riera J, Torres F, Masclans JR. High-flow oxygen therapy in acute respiratory failure.Respiratory care. 2010;55(4):408-413. 
27. Hasani A, Chapman TH, McCool D, Smith RE, Dilworth JP, Agnew JE. Domiciliary humidification improves lung mucociliary clearance in patients with bronchiectasis. Chronic respiratory disease. 2008;5(2):8186.

28. Spence KL, Murphy D, Kilian C, McGonigle R, Kilani RA. High-flow nasal cannula as a device to provide continuous positive airway pressure in infants. Journal of perinatology : official journal of the California Perinatal Association. 2007;27(12):772-775.

29. Wilkinson DJ, Andersen CC, Smith K, Holberton J. Pharyngeal pressure with high-flow nasal cannulae in premature infants. Journal of perinatology : official journal of the California Perinatal Association. $2008 ; 28(1): 42-47$.

\section{Hosted file}

Table 1.pdf available at https://authorea.com/users/402644/articles/514447-high-flownasal-cannula-versus-non-invasive-ventilation-in-severe-asthma-two-years-prospectiveobservational-study

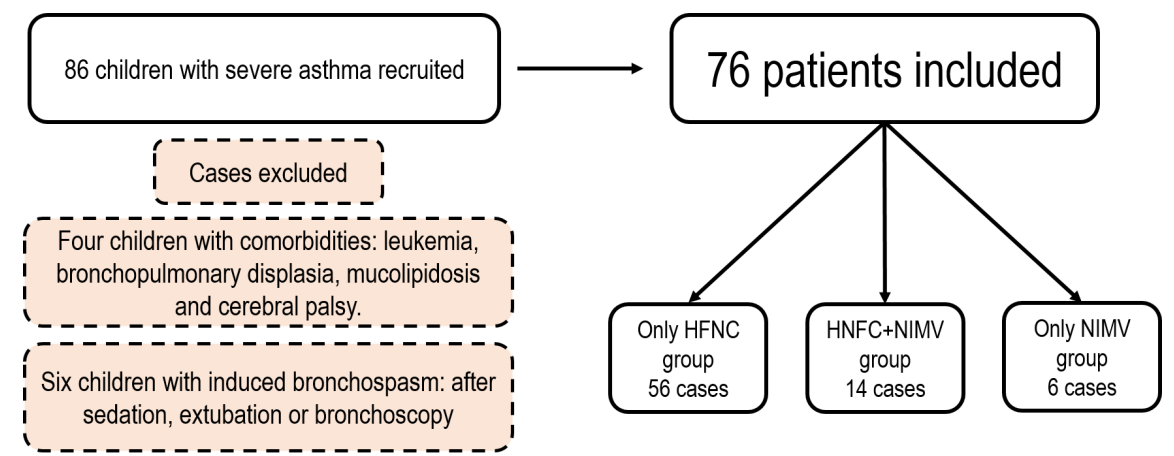

Legend: HFNC: High Flow Nassal Cannulas, NIMV: Non Invasive Mechanical Ventilation

\section{Hosted file}

Table 2.pdf available at https://authorea.com/users/402644/articles/514447-high-flownasal-cannula-versus-non-invasive-ventilation-in-severe-asthma-two-years-prospectiveobservational-study

\section{Hosted file}

Table 3.pdf available at https://authorea.com/users/402644/articles/514447-high-flownasal-cannula-versus-non-invasive-ventilation-in-severe-asthma-two-years-prospectiveobservational-study 